\title{
Radiation damage to MPPCs by irradiation with protons
}

\section{T. Matsumura*; T.Shinkawa}

Department of Applied Physics, National Defense Academy in Japan, Yokosuka 239-0811, Japan

\section{T. Hirai, K. Miyabayashi}

Department of Physics, Nara Women's University, Nara 630-8506, Japan

\section{T. Hiraiwa, A. Okamura, T. Tsunemi}

Department of Physics, Kyoto University, Kyoto 606-8502, Japan

\section{K. Horie, S. Shimizu}

Department of Physics, Osaka University, Toyonaka 560-0043, Japan

\section{Kuze, T. Matsubara}

Department of Physics, Tokyo Institute of Technology, Tokyo 152-8551, Japan

\section{T. Nakadaira, I. Nakamura}

IPNS, High Energy Accelerator Research Organization (KEK), Tsukuba 305-0801, Japan

\section{T. Nakagawa, I. Saitoh}

Nuclear Professional School, The University of Tokyo, Tokai 319-1188, Japan

\section{T. Sawada, M. Yosoi}

Research Center for Nuclear Physics, Osaka University, Ibaraki 567-0047, Japan

\section{S. Tsunoda}

Department of Physics, Tokyo University of Science, Tokyo 162-8601, Japan

Radiation damage of Multi-Pixel Photon Counters (MPPCs) caused by proton and neutron irradiation was evaluated. An increase of the leakage current, which is an indication of radiation damage on silicon devices, was observed after both the proton and neutron irradiation. Darknoise rates significantly increased due to the damage and this effect resulted in the loss of the photon counting capability because of the noise pile-up. From the leakage current measurement we found the damage level of the MPPCs caused by the neutron influence of $10^{9} \mathrm{n} / \mathrm{mm}^{2}$ is almost same level as the case of 20 Gy proton irradiation.

International workshop on new photon-detectors PD07

June 27-29 2007

Kobe University, Kobe, Japan

\footnotetext{
* Speaker.

†E-Mail:toru@nda.ac.jp
} 


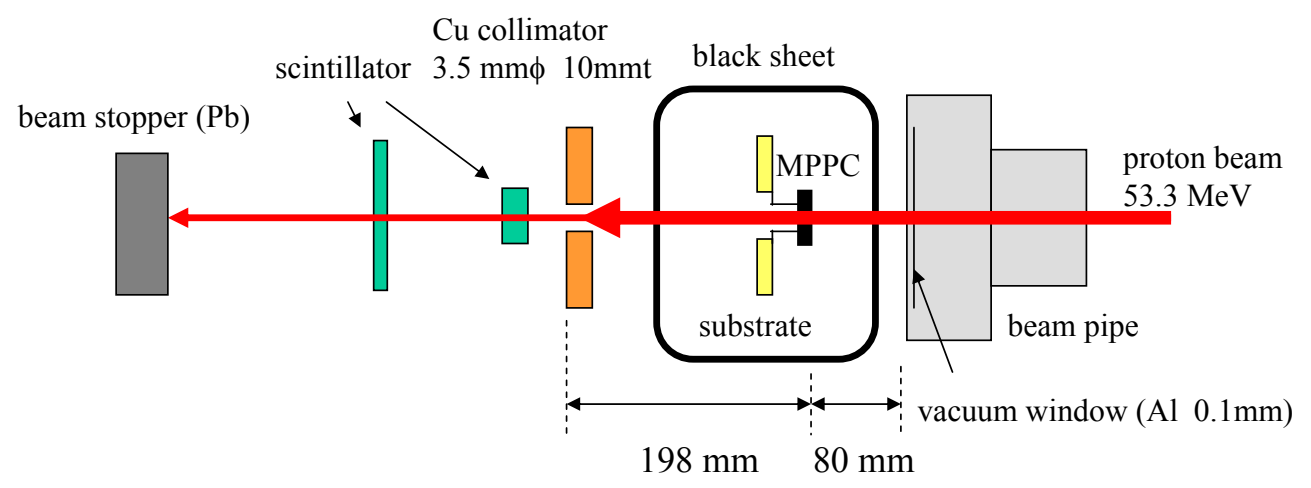

Figure 1: Setup for the proton irradiation.

\section{Introduction}

Pixelized Photon Detector (PPD), a generic name of Multi-Pixel Geiger-mode APDs, is a recently developed high sensitivity photon-detector. This device has several great advantages over photo-multiplier tubes (PMT) such as compactness, low bias voltage, low power consumption, insensitive to magnetic field and so on, although the active area of the sensor is limited to be $1 \sim 25$ $\mathrm{mm}^{2}$ [1]. Therefore this device is very useful for high energy physics, space science experiments as well as medical applications, especially to readout scintillating fibers, wave length shifting fibers, and fine granulated scintillating crystals, for example.

For a practical use of the PPDs, radiation hardness is one of the important properties to be investigated. Hence, we (members of KEK Detector Technology Project) have performed a series of radiation hardness tests of Multi-Pixel Photon Counters (MPPCs), which is one of PPDs developed by Hamamatsu Photonics, with different radiation sources, such as protons, neutrons and $\gamma$-ray[2]. The purpose of these studies are to evaluate effects caused by radiation damage on basic performance of the MPPCs. In this report, we discuss the experimental results of the proton and neutron irradiation.

\section{Proton irradiation}

\subsection{Experiment}

Two MPPC samples (labelled as \#20 and \#21) were used for the experiment. Both are the 400 pixels type MPPC, S10362-11-050C, which were delivered in February 2007.

We used a proton beam obtained from the AVF cyclotron at Research Center for Nuclear Physics of Osaka University for the irradiation, where the beam energy was $53.3 \mathrm{MeV}$. The beam was shaped to be a rectangular having a size of $8 \times 6 \mathrm{~mm}^{2}$, which was large enough to cover the active area of the MPPC $\left(1 \times 1 \mathrm{~mm}^{2}\right)$. The distribution of the beam intensity around the beam center was almost flat; the uniformity of the beam intensity in the region of $1 \times 1 \mathrm{~mm}^{2}$ was less than $5 \%$.

Figure 1 shows the experimental setup for the proton irradiation. One of the MPPC samples, which was covered with a black sheet for light tightness, was placed just after the beam-pipe flange. 

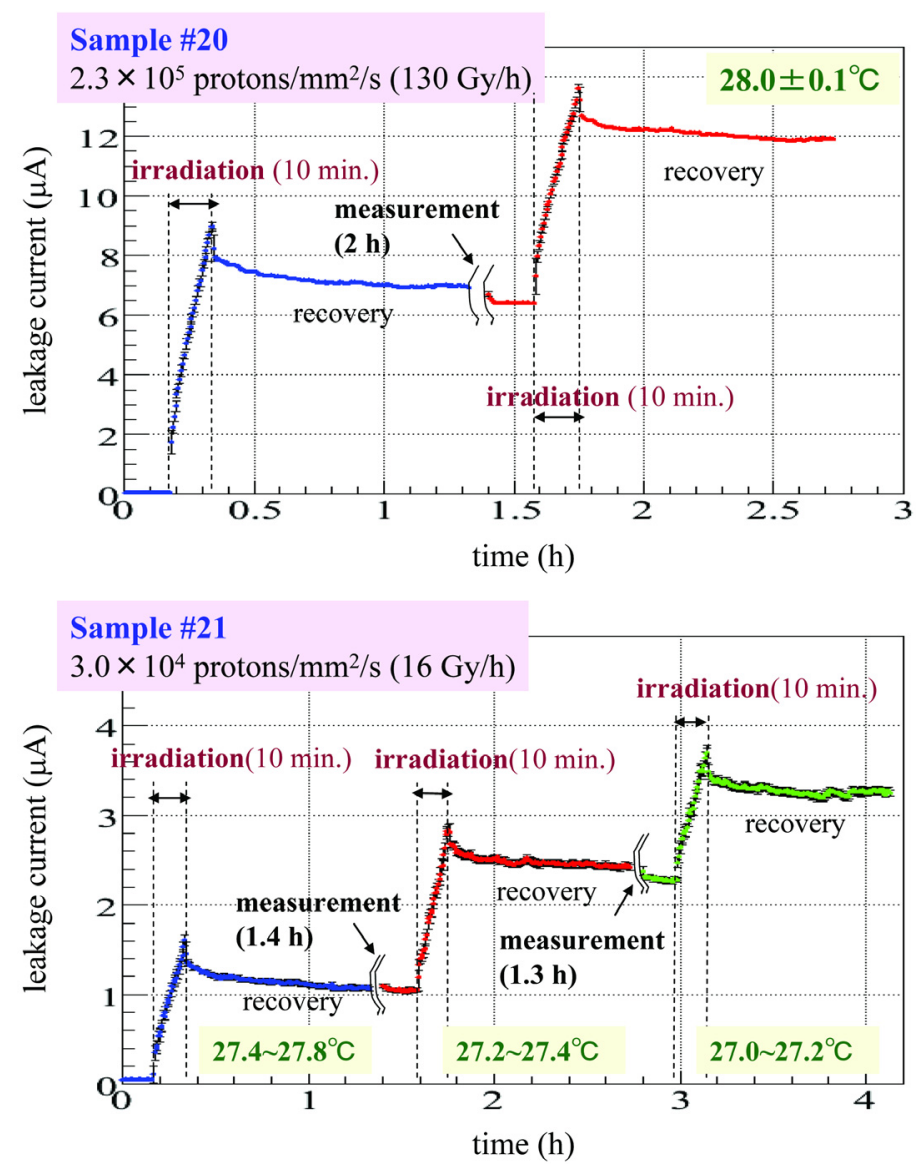

Figure 2: Variation of the leakage current for sample \#20 (top) and sample \#21 (bottom).

In order to hold the MPPC sample in the beam spot, it was mounted on a print substrate having a hole to avoid its radio activation. The proton beam was injected onto the MPPC surface. We placed a copper collimator having a hole of $3.5 \mathrm{~mm} \phi$, where the hole size was determined to have the uniform flux region of the beam spot. The beam intensity was monitored with two plastic scintillators installed downstream from the collimator.

We irradiated the MPPC samples with different beam flux. One of the MPPC, sample \#20, was irradiated with $2.3 \times 10^{5}$ protons $/ \mathrm{mm}^{2} / \mathrm{s}$, corresponding to $130 \mathrm{~Gy} / \mathrm{h}$ in dose rate. The other MPPC, sample \#21, was irradiated with lower flux, $3.0 \times 10^{4}$ protons $/ \mathrm{mm}^{2} / \mathrm{s}$, corresponding to 16 $\mathrm{Gy} / \mathrm{h}$ in dose rate. The total irradiation time was 20 minutes for sample \#20 and 30 minutes for sample \#21, respectively. Hence, the total fluence (dose) was $2.8 \times 10^{8}$ protons $/ \mathrm{mm}^{2}$ (42 Gy) for sample \#20 and $5.4 \times 10^{7}$ protons $/ \mathrm{mm}^{2}$ (8.0 Gy) for sample \#21.

\subsection{Result}

The upper plot in Fig. 2 shows the variations of the leakage current as a function of time during the experiment for the sample \#20. At the beginning, the leakage current was quite low $(\sim 0.05 \mu \mathrm{A})$; however, during the irradiation the leakage current increased linearly with time due 


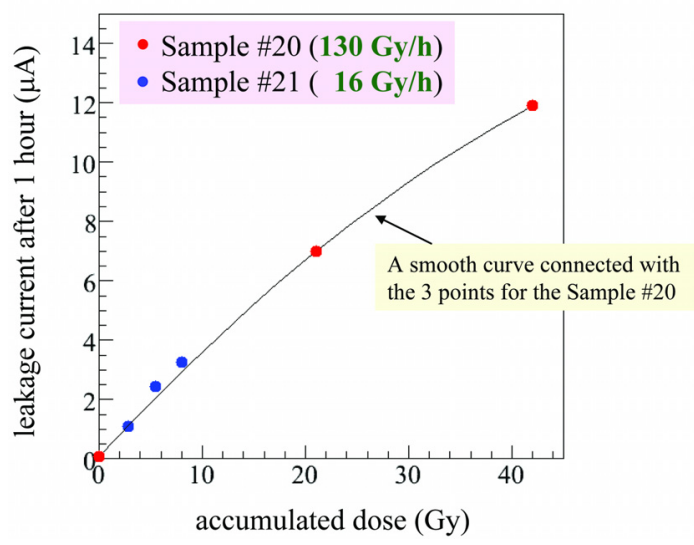

Figure 3: Relation between the leakage current and accumulated dose for both samples.
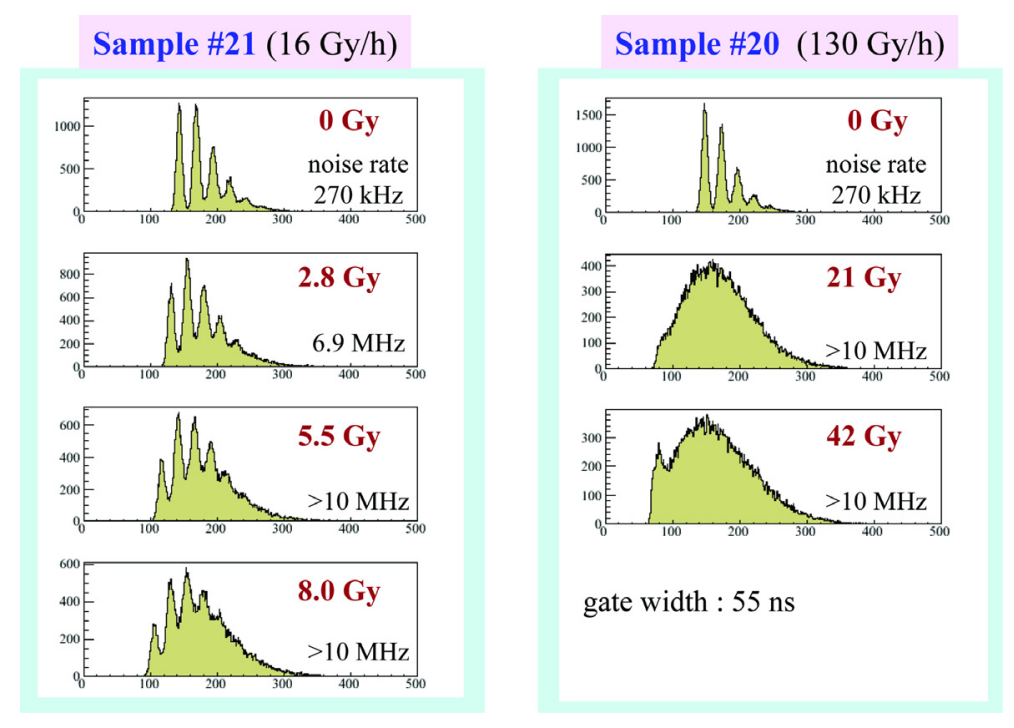

Figure 4: ADC distributions of the MPPC samples before and after the irradiation.

to radiation damage. We stopped the beam after the 10 minutes of irradiation. The leakage current gradually decreased after the beam stop, which indicates that the radiation damage caused by the proton irradiation is recovered somewhat although the recovery effect seems to be not completed within a few hours. For sample \#21, which irradiated with lower beam-flux, the variation shows the same tendency as the sample \#20 except for the increasing rate (see the bottom plot in Fig.2).

The relation of the leakage current and the accumulated dose for the both samples is plotted in Fig.3. The leakage current increase is described by an universal curve as a function of radiation dose between different dose rate. This means that the increase of the leakage current, which is caused by radiation damage due to the proton beam, is independent of the irradiation intensity.

Figure 4 shows ADC distributions of the MPPC samples before and after the irradiation, where a blue LED was flashed in synchronization with $100 \mathrm{~Hz}$ gate signals having a width of $55 \mathrm{~ns}$. Before 


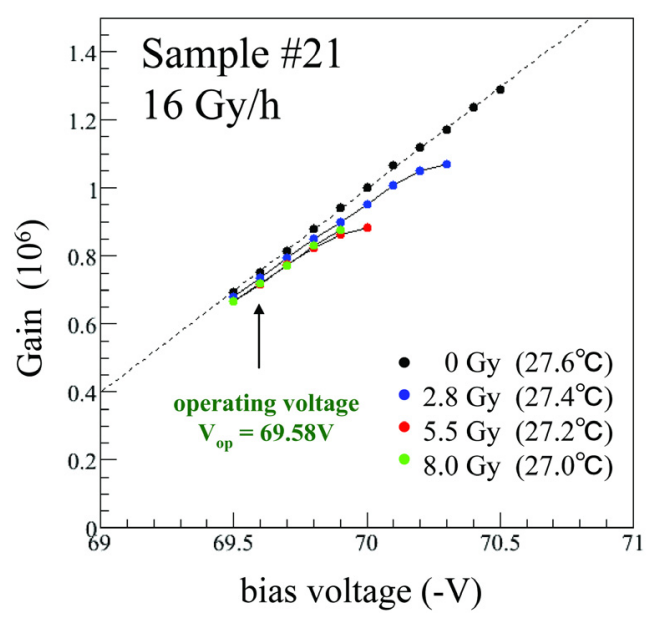

Figure 5: MPPC gain as a function of the bias voltages for different irradiated doses.

the irradiation (top histograms) the distributions of both samples clearly show the peak structure corresponding to pedestal, 1 p.e., 2 p.e., etc.. However, the peak structure is smeared due to noise pile-up after the proton irradiation. In fact, the dark-noise rate is significantly increased from 270 $\mathrm{kHz}$ to $6.9 \mathrm{MHz}$ after the $2.8 \mathrm{~Gy}$ irradiation. No peak is observed in the distributions for more than 21 Gy because of the heavy baseline fluctuations caused by noise pile-up. Therefore, the MPPCs irradiated with a certain amounts of protons $(8 \sim 21 \mathrm{~Gy})$ lose the photon counting capability due to the radiation damage effects.

The gain of MPPCs was measured before and after the irradiation. The result of the gain measurement is shown in Fig.5 as a function of bias voltage. Before the irradiation, the plot shows linear dependence reflecting the relation $G=(C / e)\left(V-V_{0}\right)$, where $G, C, V_{0}$ and $e$ are gain, pixel capacitance, break-down voltage of the MPPC and the electron charge, respectively. This linear dependence seems to be broken after the irradiation, especially at higher bias voltages. The reason is that the bias voltages are effectively dropped at resisters used in a filter circuit. However, at the operating voltage $(-69.58 \mathrm{~V})$, no significant gain drop was observed up to $8 \mathrm{~Gy}$; it was less than $5 \%$.

\section{Neutron irradiation}

\subsection{Experiment}

The experiment of neutron irradiation was performed with the fast neutron source reactor of the University of Tokyo (YAYOI). The energy range of the neutron source is from 0 to $10 \mathrm{MeV}$, where the dominant contribution of the energies is around 0.1-1 MeV. Two types of MPPC, S1036211-100CK(100 pixels) and S10362-11-050CK(400 pixels), were prepared for the irradiation. We mounted the MPPC samples inside a light shielding plastic capsule and then inserted it into the reactor. The neutron fluence was adjusted by the reactor power and the exposure time. Total fluence for each sample was estimated to be $0.84 \times 10^{6} \mathrm{n} / \mathrm{mm}^{2}, 1.7 \times 10^{6} \mathrm{n} / \mathrm{mm}^{2}, 2.6 \times 10^{6} \mathrm{n} / \mathrm{mm}^{2}$, 


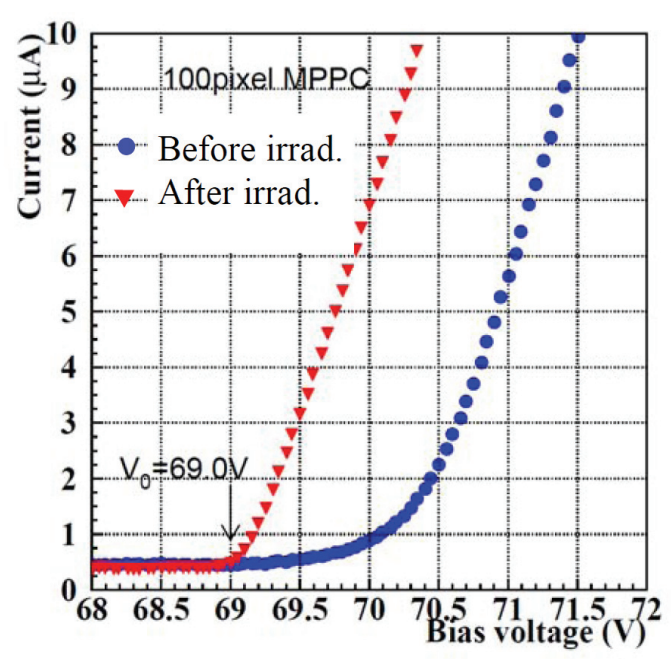

Figure 6: $I-V$ curves of the MPPC samples before and after the $1.0 \times 10^{9} \mathrm{n} / \mathrm{mm}^{2}$ irradiation.

$3.4 \times 10^{6} \mathrm{n} / \mathrm{mm}^{2}$ and, $1.0 \times 10^{9} \mathrm{n} / \mathrm{mm}^{2}, 1.0 \times 10^{10} \mathrm{n} / \mathrm{mm}^{2}, 1.0 \times 10^{11} \mathrm{n} / \mathrm{mm}^{2}$, respectively ${ }^{1}$. In this experiment, we evaluated performance degradation of the MPPC samples due to radiation damage by checking the leakage current, noise rate and variation of the breakdown voltage.

\subsection{Result}

The leakage currents were measured before and after the neutron irradiation. We found a drastic increase of the leakage current after the $1.0 \times 10^{9} \mathrm{n} / \mathrm{mm}^{2}$ irradiation, although no significant change was observed up to the $3.3 \times 10^{6} \mathrm{n} / \mathrm{mm}^{2}$. Figure 6 shows the $I-V$ curve for the samples irradiated with $1.0 \times 10^{9} \mathrm{n} / \mathrm{mm}^{2}$. As one can see, the leakage current increased from the original values in the region above the breakdown voltage $(69.0 \mathrm{~V})$. The neutron fluence of $1.0 \times 10^{8} \mathrm{n} / \mathrm{mm}^{2}$ is found to give a similar damage to $\sim 20$ Gy by proton irradiation.

The output pulses generated from the samples were monitored by an oscilloscope. We observed continuous pulse-hight signals after the $1.0 \times 10^{9} \mathrm{n} / \mathrm{mm}^{2}$ irradiation. This indicates that the photon counting capability of the samples is lost due to radiation damage.

Variations of noise rates and breakdown voltage were checked for the samples exposed to the neutron fluence up to $3.3 \times 10^{6} \mathrm{n} / \mathrm{mm}^{2}$ where the irradiated MPPCs still have photon counting capability. The result shows no significant change on the noise rate and breakdown voltage in such amount of neutron dose.

\section{Summary}

The effects of radiation damage on Multi-Pixel Photon-Counters were investigated with the proton and the neutron sources. The leakage current of the MPPC samples increased after the irradiation in both cases. This is an indication of radiation damage of MPPC. The photon counting

\footnotetext{
${ }^{1}$ The neutron fluence was re-estimated after the PD07 workshop. According to the re-estimation, we found that the neutron fluence presented in the workshop was under-estimated about one order of magnitude. The estimation has been modified in this report.
} 
capability of MPPCs seems to be lost at a certain damage level. From the leakage current measurement we found the damage level of the MPPCs caused by the neutron influence of $10^{9} \mathrm{n} / \mathrm{mm}^{2}$ is almost same level as the case of 20 Gy proton irradiation.

\section{Acknowledgement}

The present work was supported in part by the Ministry of Education, Science, Sports and Culture of Japan with the Grant-in-Aid for Scientific Research No. 17204020.

\section{References}

[1] D. Renker, Nucl. Instrum. Meth. A 567, 48 (2006).

[2] T. Matsubara, in these proceedings. 